\title{
Effect of External Magnetic Field on Compact Inductively-coupled Reactive Ion Etching Reactor
}

\author{
Taisei MOTOMURA*1, Kazunori TAKAHASHI*2, \\ Yuji KASASHIMA*1 ${ }^{*}$ Fumihiko UESUGI*1 and Akira ANDO*2 \\ ${ }^{*}$ Advanced Manufacturing Research Institute, National Institute of Advanced Industrial Science and Technology, \\ 807-1 Shuku-machi, Tosu-shi, Saga 841-0052, Japan, \\ ${ }^{* 2}$ Department of Electrical Engineering, Tohoku University, 6-6-05 Aramaki-Aza-Aoba, Aoba-ku, Sendai-shi, Miyagi 980-8579, Japan
}

(Received May 8, 2015, Accepted July 17, 2015)

A compact inductively-coupled plasma etching reactor with inner diameter of $38 \mathrm{~mm}$ without diffusion chamber (i.e., chamber diameter of plasma etching area is the same as that of plasma discharge area) has been developed. An external magnetic field, created by a solenoid current of $\gtrsim 10 \mathrm{~A}$ that means magnetic field strength of $\gtrsim 0.02 \mathrm{~T}$ at the center of solenoid coil, can effectively reduce losses of plasma at the chamber wall. At low RF power of around $50 \mathrm{~W}$, a discharge-mode transited from capacitively- to inductively-coupled plasmas, high electron density plasma was generated and the optical emissions of fluorine increased in intensity. In summary, the external magnetic field maintains the high plasma density and the compact reactor for a processing area as small as $10 \mathrm{~mm}$ diameter has been demonstrated. The basic etching characteristics were evaluated in the case of a Si wafer masked with a $\mathrm{SiO}_{2}$ film. Typical etching rate of $\gtrsim 0.3 \mu \mathrm{m} / \mathrm{min}$ was obtained at conditions with a solenoid current of $30 \mathrm{~A}$, a RF power of $500 \mathrm{~W}$, a pulsed plasma discharge with duty ratio of $10 \%$, and a chamber pressure at $0.2 \mathrm{~Pa}$.

\section{Introduction}

Over the past few decades, silicon processes using large diameter wafers have advanced, in order to reduce the cost of the semiconductor manufacturing and achieve economic benefits. At the same time, larger diameter plasma etching reactors have been developed, with multiple discharge modes capable of producing both capacitively-coupled plasma (CCP) and inductively-coupled plasma (ICP) ${ }^{1,2)}$. The presence of an external magnetic field, and a suitable choice of a driving frequency and plasma source geometry, are well known to improve the plasma production efficiency via wave-plasma coupling (e.g., electron cyclotron resonance (ECR) plasma $^{3,4)}$ and a helicon wave plasma ${ }^{5-8)}$ ). In conventional reactors, an efficient high-density plasma source is connected to a larger diameter diffusion chamber, and expanded by an external magnetic field, in order to treat large diameter silicon wafers ${ }^{9-13)}$. In silicon etching using fluorinated gases, a critical physical quantity determining the etching rate is the density of the fluorine radicals, which is roughly proportional to the electron density in weakly ionized plasmas ${ }^{14)}$. The use of larger diameter diffusion chambers reduces the ratio of the loss surface to the ionization volume, and results in high-density plasma production since the power balance between the production and the loss from the system that is a critical factor in determining the plasma density. By incorporating such geometrical configurations into etching reactors, high-speed plasma process has been achieved using diffusion chambers ${ }^{10-12)}$. Compact plasma processing reactors can be especially utilized for the research and development ( $R$ \& D) in fields such as material and microelectromechanical system (MEMS) research because an installation cost of reactor and development cost of devices can be reduced. Such compact plasma etching reactors, however, have small diameter vacuum chambers that cause significant plasma loss to the chamber wall, which results in low plasma density, and thereby a low etching rate, especially in etching reactors. The technological challenge in the case of such reactors lies in the fact that the ratio of the loss surface to the ionization volume increases when the size of the reactor is reduced, meaning that efficient plasma production is difficult.

Maintaining sufficient plasma density is necessary to obtain a desirable etching rate in reactive ion etching $(\mathrm{RIE})^{15)}$. The question, then, is how to do so using a compact etching reactor. In order to create a compact RF plasma etching reactor without diffusion chamber, the application of an external magnetic field is proposed, to reduce the plasma loss to the side wall of the small diameter vacuum chamber. High density plasma production $\left(\sim 10^{18} \mathrm{~m}^{-3}\right)$ under low gas-pressure operation $(\sim 0.1 \mathrm{~Pa})$ is required to obtain a collisionless sheath for ions and resulting high aspect ratio RIE. In summary, efficient plasma production in a low-pressure compact reactor without diffusion chamber is required to develop a compact RIE device.

In the present study, the plasma production characteristics of a compact ICP reactor with an external magnetic field were experimentally investigated for various magnetic field strengths, using $\mathrm{Ar}$ and $\mathrm{SF}_{6}$ gases. In the case of Ar, electron density was measured using a Langmuir probe (Section 3.1); while, in the case of $\mathrm{SF}_{6}$, the optical emission intensity of the fluorine radical was investigated to assess the relative value of the fluorine radical in silicon etching (Section 3.2). A significant increase in the plasma density in the case of Ar, and emission intensity in the case of $\mathrm{SF}_{6}$, was detected when the magnetic field strength was increased to $0.02 \mathrm{~T}$, which implies a discharge-mode transition from $\mathrm{CCP}$ to $\mathrm{ICP}$ as a result of the inhibition of plasma loss to the chamber wall. In ad- 
dition, a silicon etching process based on the greater emission intensity for $\mathrm{SF}_{6}$ was preliminarily tested (Section 3.3)

\section{Experimental methods}

Figure 1 shows a schematic diagram of the proposed RIE reactor. A Pyrex glass tube with an inner diameter of $36 \mathrm{~mm}$ and a length of $600 \mathrm{~mm}$ was connected to a grounded stainless steel (SUS) chamber (not shown in Fig. 1), which was evacuated using a turbomolecular and a rotary pump system to a base pressure of $\sim 5 \times 10^{-4}$ $\mathrm{Pa}$. A water-cooled solenoid coil was centered at the axial location of $z=108 \mathrm{~mm}$ to produce an external DC
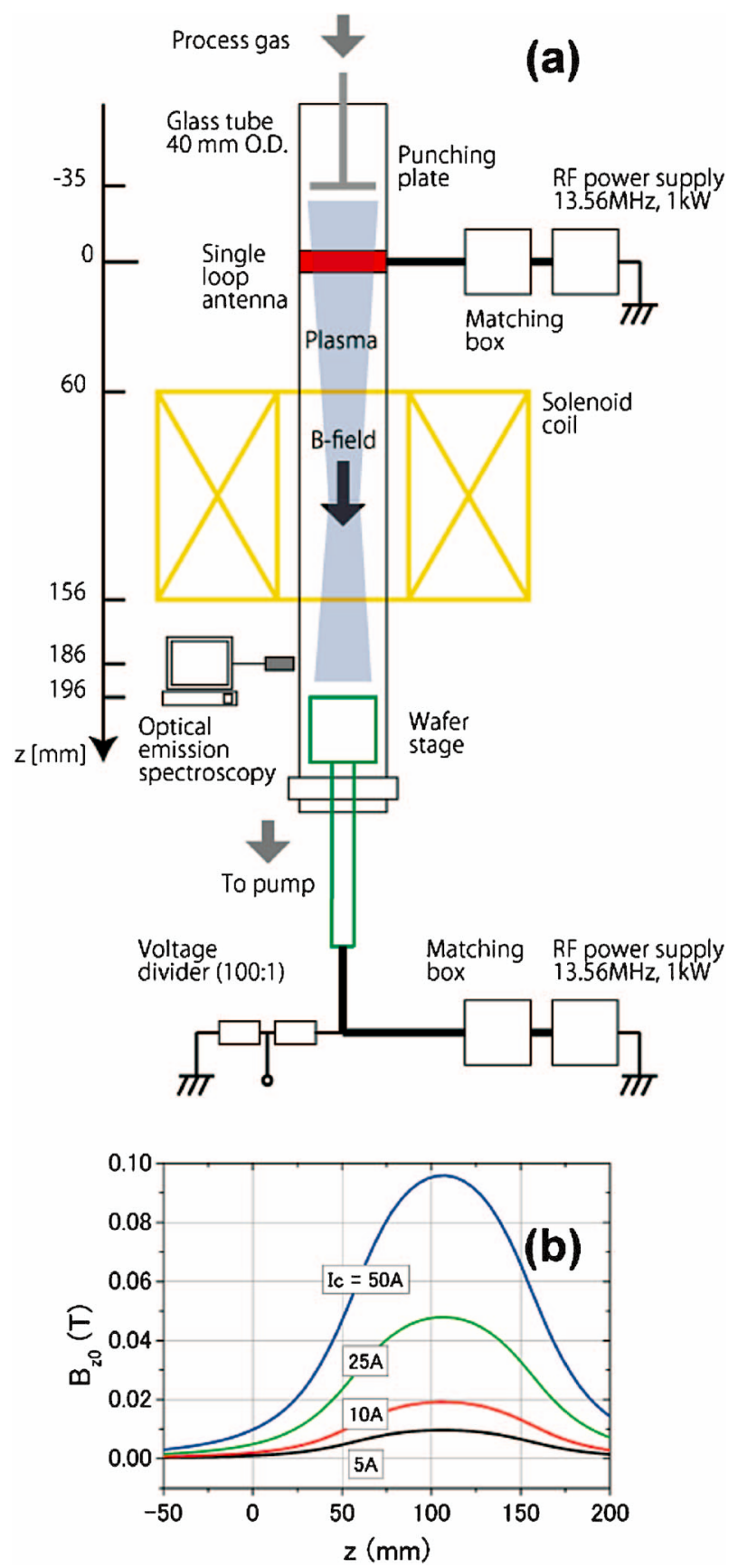

Fig. 1 Experimental configuration. (a) Schematic drawing of the experimental setup. (b) Axial profiles of the background DC magnetic field along the $z$-axis, $B_{z 0}$, for various values of the coil current $I_{\mathrm{c}}$. magnetic field, where $z=0$ was defined as the location of an RF antenna (described later). The magnetic field configuration using the solenoid coil could be varied by controlling the coil current $I_{c}$, as shown in Fig. 1(b). A single-loop RF antenna for plasma production was wound around the glass tube at $z=0 \mathrm{~mm}$, and connected to an RF power supply, with $13.56 \mathrm{MHz}$ excitation frequency, through a matching box consisting of two variable capacitors. The RF power for the plasma production (input RF power $P_{\text {in }}$ ) was pulsed with a duration time of $100 \mathrm{~ms}$ and repetition rate of $1 \mathrm{~Hz}$, and with a duty ratio of $10 \%$. In this experiment, we used pulsed RF power operation because of the low thermal resistance of the Pyrex glass tube. Either $\mathrm{SF}_{6}$ or Ar was introduced from the top of the glass tube. A mass flow controller maintained the mass flow rate of $\mathrm{SF}_{6}$ at $8.0 \mathrm{sccm}$, while a needle valve controlled the mass flow rate of Ar gas (flow not measured). The gas flow rates were chosen so as to maintain a chamber pressure of $0.2 \mathrm{~Pa}$ in both cases, with the steady gas pressure measured with an ionization gauge connected to a side port of the grounded SUS chamber. A grounded SUS punching plate was placed at $z=-35 \mathrm{~mm}$, which terminated an effective plasma column. The wafer stage had a mechanical chucking system for holding a $12.5 \mathrm{~mm}$ diameter wafer, and was electrically connected to another RF power supply, through a matching box, in order to apply self-bias voltage. The top edge of the wafer stage was placed at $z=196 \mathrm{~mm}$. The input power to the wafer stage was fixed at $20 \mathrm{~W}$ (continuous operation) with a $13.56 \mathrm{MHz}$ excitation frequency. When the input RF power was greater than 50 $\mathrm{W}$, a pulsed ICP discharge using the RF antenna, and a continuous CCP discharge using the wafer stage, occurred at the same time, where the RF power supply for the self-bias could unfortunately not be pulsed. A voltage divider (100:1) was connected to the wafer stage to measure the self-bias voltage, and the self-bias voltage remained at a near constant level of approximately -150 $\mathrm{V}$ through experiments. When the input RF power $P_{\text {in }}$ was $0 \mathrm{~W}$, however, the self-bias voltage was approximately $-80 \mathrm{~V}$ induced by continuous operation of the input power to the wafer stage (i.e., CCP discharge with $20 \mathrm{~W}$ using only wafer stage). As described later (Section 3), the local radical density observed during only $\mathrm{CCP}$-only discharge was much less than that observed during a combination of the ICP and CCP modes, and thus the etching process involving $\mathrm{CCP}$-only discharge was considered to be negligible.

The forward and reflected RF powers, $P_{\mathrm{f}}$ and $P_{\mathrm{r}}$, were measured using directional couplers inserted between the RF power supply and the matching box for both the plasma source and the wafer stage. The input RF power to the load, including both the circuit and plasma resistance $P_{\text {in }}$, was estimated as $P_{\mathrm{f}}-P_{\mathrm{r}}$. The ratio of reflected RF power to forward RF power, $P_{\mathrm{r}} / P_{\mathrm{f}}$, was typically much less than 0.1 for both the RF systems. The electron density in the Ar plasma was estimated using a Langmuir probe placed at $z=186 \mathrm{~mm}$ and radial position $x=0 \mathrm{~mm}$, with a measured electron temperature of $4.5 \mathrm{eV}$ being used for electron density estimation. The 
ion saturation current was measured at the probe voltage of $-70 \mathrm{~V}$ for electron density estimation. Electron density measurement was only performed for the Ar plasma, because in the case of $\mathrm{SF}_{6}$, the tip of Langmuir probe (platinum wire of $\phi 0.5 \mathrm{~mm}$ and length $3 \mathrm{~mm}$ ) was etched by the RIE. To estimate the number of intermediate products of the gas phase reaction during RIE using $\mathrm{SF}_{6}$ plasma, optical emission spectroscopy was employed to monitor the time dependence of the fluorine line FI at $703.7 \mathrm{~nm}$ (excitation threshold of $14.7 \mathrm{eV})^{16-18}$. The fiber head was placed at $z=186 \mathrm{~mm}$, and the detected light was guided to a spectrometer (HR2000+, Ocean Optics) with a wavelength range of $200-1100 \mathrm{~nm}$ and wavelength resolution of $1.10 \mathrm{~nm}$. The emission intensity integration time was $50 \mathrm{~ms}$. The measured ion saturation current and emission intensity were averaged over 30 seconds operation time (i.e., averaging time is 3 seconds because duty ratio of $10 \%$ ). A $\mathrm{Si}$ wafer with a $\mathrm{SiO}_{2}$ mask was used for the RIE test. The dotted $\mathrm{SiO}_{2}$ mask was $\sim 5 \mu \mathrm{m}$ in diameter, with a mask thickness of $\sim 1 \mu \mathrm{m}$, and the etching shape was imaged using a scanning electron microscopy (SEM, S-4300, Hitachi).

\section{Results and discussion}

\subsection{Ar plasmas}

Before testing the $\mathrm{SF}_{6}$ plasmas, the basic properties of the source operation were investigated using Ar, where the electron density $n_{\mathrm{e}}$ was measured as a function of the solenoid current $I_{\mathrm{c}}$ and input RF power $P_{\text {in }}$. Figure 2 shows $n_{\mathrm{e}}$ as a function of (a) the $I_{\mathrm{c}}$ for $P_{\text {in }} \sim 500 \mathrm{~W}$ (duty ratio of $10 \%$ ), and (b) the $P_{\text {in }}$ for $I_{\mathrm{c}}=30 \mathrm{~A}$. All data were measured with an input RF power to the wafer stage of $\sim 20 \mathrm{~W}$ (continuous operation). The inset graph in Fig. 2(b) shows a I-V curve obtained by Langmuir probe measurement at $P_{\text {in }} \sim 500 \mathrm{~W}$. The ion saturation current was measured at the probe voltage of $-70 \mathrm{~V}$ for electron density estimation. The $n_{\mathrm{e}}$ was found to increase in the range of $I_{\mathrm{c}}=8-50 \mathrm{~A}$ as shown in Fig. 2(a), with the density increase around $I_{\mathrm{c}} \sim 10 \mathrm{~A}$ seemingly more conspicuous in comparison to the rest of the $I_{\mathrm{c}}$ range ( $\left.\sim 10-50 \mathrm{~A}\right)$. The magnetic field strength for $I_{\mathrm{c}}=10 \mathrm{~A}$ resulted in an ion Larmour radius of $\sim 22 \mathrm{~mm}$ (the ion temperature was assumed to be $T_{\mathrm{i}}=0.45 \mathrm{eV}$ and the magnetic field strength $B=0.02 \mathrm{~T}$ ), which was very similar to the chamber radius. The rapid increase in the plasma density implies an inhibition of plasma cross-field transport and resultant loss to the side wall. On increasing the magnetic field strength further (in the range of $I_{\mathrm{c}}=10-50 \mathrm{~A}$ ), radial plasma confinement was found to be further improved, even in the small diameter chamber. In the present experimental setup, $I_{\mathrm{c}} \gtrsim 10 \mathrm{~A}$ was required for efficient transport of the plasma to the wafer location at $z=196 \mathrm{~mm}$. In the range of $I_{\mathrm{c}}<8 \mathrm{~A}$, the $n_{\mathrm{e}}$ obtained at $z$ $=196 \mathrm{~mm}$ was so low as to be unmeasurable, because the plasma was extinguished through extensive large plasma loss to the side wall of the vacuum chamber. This occured only when the Langmuir probe was located near the $z$-axis (i.e., radial position $r=0$ ), and was probably due to the disturbance of the plasma by the probe, which may have reduced the plasma density in the small di-
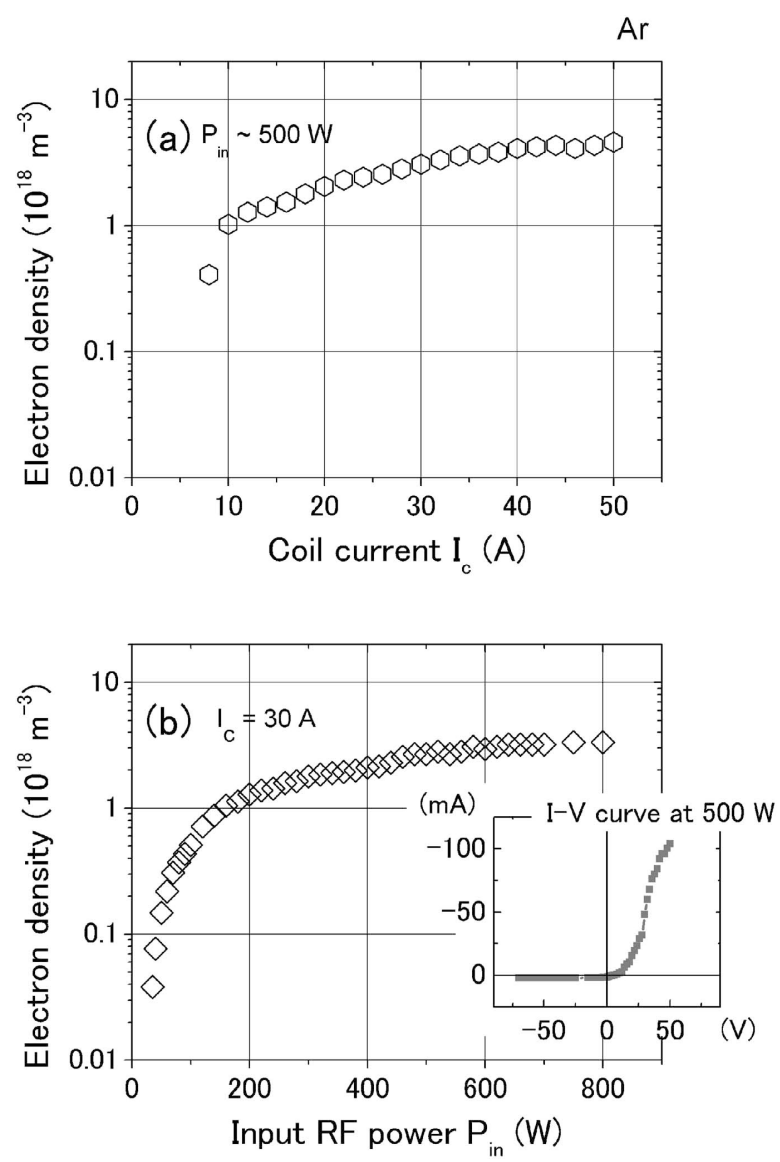

Fig. 2 Electron density as a function of (a) the coil current $I_{\mathrm{c}}$, and (b) the input RF power $P_{\text {in }}$. The experimental conditions are (a) $P_{\text {in }} \sim 500 \mathrm{~W}$ (duty ratio of $10 \%$ ) and (b) $I_{\mathrm{c}}=30 \mathrm{~A}$. All data are measured with input RF power to the wafer stage of $\sim 20 \mathrm{~W}$ (continuous operation) in Ar plasmas. Inset graph in Fig. 2(b) is I-V curve obtained by Langmuir probe measurement at $P_{\text {in }} \sim 500 \mathrm{~W}$. The ion saturation current was measured at the probe voltage of $-70 \mathrm{~V}$ for electron density estimation.

ameter chamber because of the large sheath length around the probe tip in comparison with the chamber volume.

As shown in Fig. 2(b), $n_{\mathrm{e}}$ increased with an increase in $P_{\text {in }}$, similar to the results in Refs. 19 and 20. A sharp rise in $n_{\mathrm{e}}$ was observed at $\gtrsim 30 \mathrm{~W}$ [Fig. 2(b)], probably due to the transition in the discharge mode from CCP to ICP as in the basic plasma production experiment performed by Degeling et $\mathrm{al}^{7}$ ). The $n_{\mathrm{e}}$ increased very gradually in the region of $P_{\text {in }} \gtrsim 100 \mathrm{~W}$ because the discharge mode remained stable in ICP discharge mode and the skin depth of the electromagnetic field was reduced by the higher density plasma. There was no clear presence of the helicon wave in the present setup, and the plasma appeared to be mainly produced by the ICP mode discharge. Typically, a B-dot probe is used to simply measure an excited RF magnetic field along the $z$-axis (i.e., an axial profile of the helicon wave), to approve production of helicon plasma ${ }^{21,22)}$. In this experiment, however, there was no axial access port for installing a B-dot probe, and thus this issue must remain for future study. The electron temperature was evaluated by using I-V curve shown by inset graph in Fig. 2(b) and is slightly 
high $(\sim 4.5 \mathrm{eV})$ in comparison to that of typically laboratory plasma experiment $(\sim 3 \mathrm{eV})$. This is probably due to the large plasma loss caused by the use of small diameter vacuum chamber and high-energy electrons in RF plasma discharge; however, these issues also remain for future study. Figure 2 shows both the decrease in plasma loss to the side wall as a result of efficient plasma transport [Fig. 2(a)], and efficient plasma production by means of ICP discharge [Fig. 2(b)].

\section{2 $\quad \mathrm{SF}_{6}$ plasmas}

Measurements of the emission intensity of fluorine $(703.7 \mathrm{~nm})$ from the $\mathrm{SF}_{6}$ plasmas was performed for various value of input RF power $P_{\text {in }}$ and solenoid current $I_{\mathrm{c}}$, to evaluate the fluorine radical production, which contributes to silicon etching. Figure $\mathbf{3}$ shows the relative emission intensity of fluorine $(703.7 \mathrm{~nm})$ as a function of (a) the $I_{\mathrm{c}}$ for $P_{\text {in }} \sim 500 \mathrm{~W}$ (duty ratio of $10 \%$ ), and (b) the $P_{\text {in }}$ for $I_{\mathrm{c}}=30 \mathrm{~A}$. All data were measured with an input $\mathrm{RF}$ power to the wafer stage of $\sim 20 \mathrm{~W}$ (continuous operation) in $\mathrm{SF}_{6}$ plasmas.

As shown in Fig. 3(a), the emission intensity increased by an order of magnitude with the increase in the solenoid current from $I_{\mathrm{c}} \sim 10-15 \mathrm{~A}$, similar to the increase in ion density in the case of Ar. The intensity further increased, several hundred times, from $I_{\mathrm{c}} \sim 15-30$ A, rela-
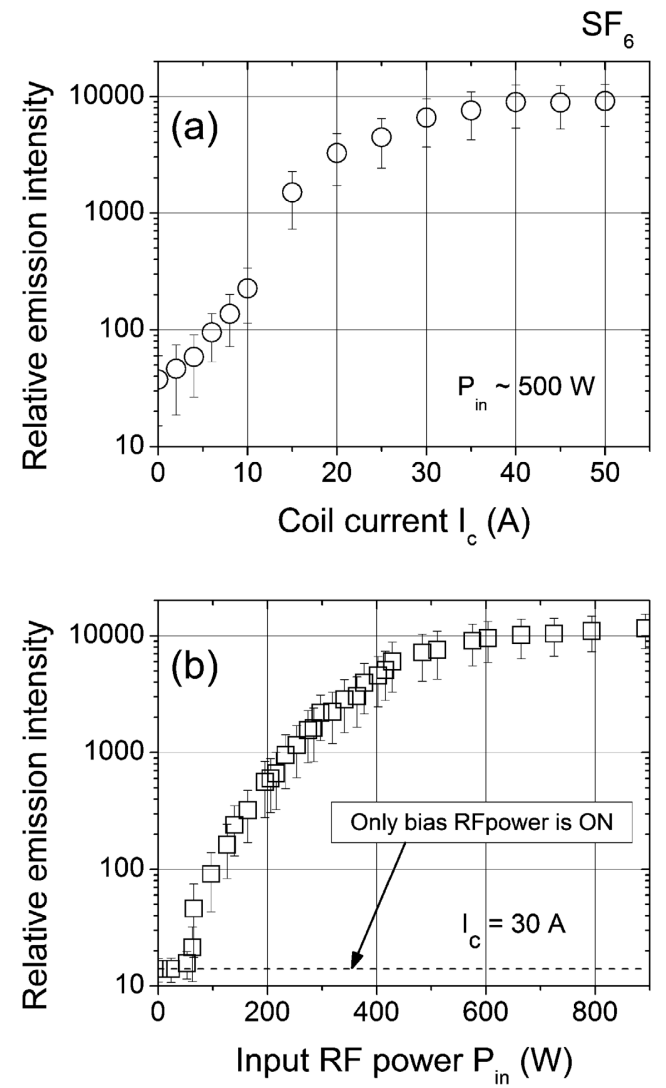

Fig. 3 Relative emission intensity as a function of (a) the coil current $I_{\mathrm{c}}$, and (b) the input RF power $P_{\text {in }}$. The experimental conditions are (a) $P_{\text {in }} \sim 500 \mathrm{~W}$ (duty ratio of $10 \%$ ), and (b) $I_{\mathrm{c}}=$ $30 \mathrm{~A}$. All data are measured with input RF power to the wafer stage of $\sim 20 \mathrm{~W}$ (continuous operation) in $\mathrm{SF}_{6}$ plasmas. The relative emission intensity is $\sim 10$ when only the bias RF power (only the CCP discharge) is $\mathrm{ON}$. tive to the no magnetic field case, and was comparatively saturated $I_{\mathrm{c}} \gtrless 40 \mathrm{~A}$. Since the magnetic field inhibited the cross-field plasma transport, as in the case of Ar, the electron density responsible for radical production was considered to be increased. Therefore, greater emission intensity from the fluorine radical, which implies greater radical density, can be obtained under stronger magnetic field conditions. Although such strong magnetic fields require significant amounts of electricity, a DC power supply, and larger solenoids, this result provides a guideline for designing a magnetic field configuration sufficient for constructing a compact etching reactor in the near future.

The dashed line in Fig. 3(b) shows the emission intensity with the RF power to the wafer stage but no input RF power to the RF antenna $\left(P_{\text {in }}=0\right)$ (i.e., only for the $\mathrm{CCP}$ discharge driven by the wafer stage). At an RF power of less than $50 \mathrm{~W}$, emission intensity data similar to that obtained for the CCP discharge was observed [Fig. 3(b)]. At an RF power of around $50 \mathrm{~W}$, the intensity rapidly increased due to the transition from CCP to ICP mode. Hence, high density plasma production through mode transition to ICP can be achieved even in $\mathrm{SF}_{6}$ at an $\mathrm{RF}$ power of $50 \mathrm{~W}$ (interestingly, a similar value to that in the Ar case). When the source RF power was increased to $500 \mathrm{~W}$, the emission intensity was three orders of magnitude greater than in the no source RF power case. Through they remain to be fully and quantitatively understood, these data suggest that the density of the fluorine radical after ICP mode transition is much greater than in the $\mathrm{CCP}$ mode case.

Based on the results shown in Figs. 2 and 3, the RIE was tested under optimal operating conditions of $P_{\text {in }}$ $\sim 500 \mathrm{~W}$ and $I_{\mathrm{c}}=30 \mathrm{~A}$, using the present experimental setup. Figure 4 shows a SEM image of the etched silicon at $P_{\text {in }}=500 \mathrm{~W}$ and $I_{\mathrm{c}}=30 \mathrm{~A}$, where a Si wafer with $\mathrm{SiO}_{2}$ mask was etched by ICP discharge over a total operation time of $30 \mathrm{~min}$ (total ICP irradiation time is $3 \mathrm{~min}$ because of a duty ratio of $10 \%$ ). The pulsed RF power was $500 \mathrm{~W}$, with a duty ratio of $10 \%$. In this case, the etching rates for the $\mathrm{Si}$ and $\mathrm{SiO}_{2}$ mask were estimated to be $\gtrsim 0.3$

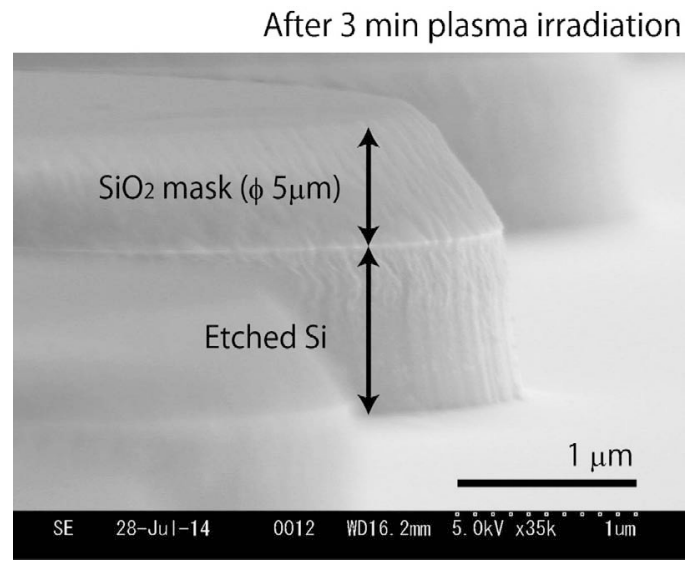

Fig. 4 SEM image after 3-min ICP irradiation. The input RF power to the plasma source is approximately $500 \mathrm{~W}$ (duty ratio of $10 \%$ ), and the coil current is $I_{\mathrm{c}}=30 \mathrm{~A}$. The input RF power to the wafer stage is $\sim 20 \mathrm{~W}$ (continuous operation). 
and $\lesssim 0.1 \mu \mathrm{m} / \mathrm{min}$, respectively, as shown in Fig. 4 . The etching depth of $\mathrm{Si}$ and $\mathrm{SiO}_{2}$ were evaluated by using SEM images after 3 min ICP irradiation. The thickness of $\mathrm{Si}$ under $\mathrm{SiO}_{2}$ mask is used for estimation of Si etching depth. Here again it should be noted that the etching process involving only the input RF power to the wafer stage (no input RF power to the RF antenna) was ignored because of the much lower intensity of the fluorine radicals. The etching selectivity, defined by the ratio of the etching depth of $\mathrm{Si}$ to that of $\mathrm{SiO}_{2}$ mask ${ }^{23)}$, was at least three under our experimental conditions. Finally, we consider low vacuum conductance in the case of such small diameter etching reactors. In the present setup, a wafer stage of $30 \mathrm{~mm}$ outer diameter was installed in a vacuum chamber of $38 \mathrm{~mm}$ inner diameter, which induced low evacuation efficiency in the etching reaction products. Based on the result of $\gtrsim 0.3 \mu \mathrm{m} / \mathrm{min}$ for $\mathrm{Si}$ etching (Fig. 4), we conclude that the Si etching reaction, and the evacuation of the reaction products, proceeded relatively well throughout the plasma processing, without undesirable reaction products; however, the evacuation efficiency will be studied in detail in our future work. Needless to say, the vacuum conductance may easily be increased by increasing the vacuum pumping speed. An experimental setup with a control system for additive gases (e.g., $\mathrm{O}_{2}$ ) will be designed in the near future, to enable control of the shape of the etching hole. In addition, a shorter pulsed plasma discharge with higher RF power, as well as a metal etching mask will be incorporated, to control the electron temperature, and to create a deep etching hole, respectively.

\section{Conclusion}

To create a compact RF plasma etching reactor, an external, solenoid coil-based magnetic field was applied in order to reduce the plasma loss to the chamber side wall in a reactor of $38 \mathrm{~mm}$ inner diameter without diffusion chamber. As a result of the external magnetic field, the electron density increased with an increase in the solenoid coil current and input RF power, similarly to the case of conventional ICP reactors. The relative emission intensity of the fluorine radical was shown to be dependent on the solenoid coil current and input RF power, enabling the construction of a small diameter RIE reactor using RF plasma production. When $\mathrm{SF}_{6}$ gas was used in the proposed compact plasma etching reactor, the optical emission intensity of fluorine radicals near the wafer also increased with an increase in magnetic field strength and input RF power. The basic etching phenomena in the case of a $\mathrm{Si}$ wafer masked with $\mathrm{SiO}_{2}$ were determined, and an etching rate of $\gtrsim 0.3 \mu \mathrm{m} / \mathrm{min}$ was obtained. Though the proposed etching reactor still possessed a large solenoid and DC power supply, the value of such a magnetic field to plasma production and the etching process in a compact etching reactor was demonstrated.

\section{Acknowledgments}

This work was partially supported by the Adaptable and Seamless Technology Transfer Program through Target-driven R \& D from JST, and a Grant-in-Aid for Young Scientists (B 26790066) from JSPS and the Hatakeyama Culture Foundation. The author wishes to acknowledge the assistance provided by Dr. Kazuya Kikunaga, Mr. Arami Saruwatari, Mr. Michihiro Inoue, Dr. Yoshiki Shimizu, Dr. Shizuka Nakano, Dr. Hisato Ogiso, Dr. Sommawan Khumpuang, Dr. Shiro Hara and Mr. Hiroyuki Tanaka of the National Institute of Advanced Industrial Science and Technology.

\section{References}

1) M. A. Lieberman and A. J. Lichtenberg: Principles of Plasma Discharges and Materials Processing 2nd Edition (Wiley, New York, 2005), Chapter 11 and 15.

2) J. Hopwood: Plasma Sources Sci. Technol., 1 (1992) 109.

3) S. Tachi, K. Tsujimoto, S. Arai and T. Kure: J. Vac. Sci. Technol. A, 9 (1991) 796.

4) W. Miyazawa, S. Tada, K. Ito, H. Saito, S. Den, Y. Hayashi, Y. Okamoto and Y. Sakamoto: Plasma Sources Sci. Technol., 5 (1996) 265.

5) R. W. Boswell: Phys. Lett. A, 33 (1970) 457.

6) F. F. Chen: Plasma Phys. Control. Fusion, 33 (1991) 339 and references therein.

7) A. W. Degeling, C. O. Jung, R. W. Boswell and A. R. Ellingboe: Phys. Plasmas, 3 (1996) 2788.

8) R. W. Boswell and F. F. Chen: IEEE Trans. Plasma Sci., 25 (1997) 1229 and references therein.

9) Y. Horiike and M. Shibagaki: Proc. of the 7th Conf. on Solid State Device, Tokyo, Japan, 1975 (1976) Suppl. p. 13.

10) A. J. Perry and R.W. Boswell: Appl. Phys. Lett., 55 (1989) 148.

11) K. Yamaya, Y. Yamaki, H. Nakanishi and S. Chichibu: Appl. Phys. Lett., 72 (1998) 235.

12) S. Aachboun and P. Ranson: J. Vac. Sci. Technol. A, 17 (1999) 2270 .

13) S. W. Hwang, Y. J. Lee, H. R. Han, J. B. Yoo and G. Y. Yeom: J. Vac. Sci. Technol. A, 17 (1999) 1211.

14) D. L. Flamm, V. M. Donnelly and J. A. Mucha: J. Appl. Phys., 52 (1981) 3633.

15) J. W. Coburn and H. F. Winters: J. Appl. Phys., 50 (1979) 3189.

16) S. B. Radovanov, B. Tomčik, Z. Lj. Petrović, and B. M. Jelenković: J. Appl. Phys., 67 (1990) 97.

17) Z. L. Petrović, F. Tochikubo, S. Kakuta and T. Makabe: J. Appl. Phys., 73 (1993) 2163.

18) K. Sasaki, Y. Kawai and K. Kadota: Appl. Phys. Lett., 70 (1997) 1375.

19) H. Y. Lee, D. W. Kim, Y. J. Sung and G. Y. Yeom: Jpn. J. Appl. Phys., 44 (2005) 1445.

20) A. Matsutani, H. Ohtsuki and F. Koyama: Jpn. J. Appl. Phys., 49 (2010) 06GH05.

21) Y. Sakawa, N. Koshikawa and T. Shoji: Plasma Sources Sci. Technol., 6 (1997) 96.

22) T. Motomura, S. Shinohara, T. Tanikawa and K. P. Shamrai: Phys. Plasmas, 19 (2012) 043504.

23) Rudolf A.H. Heinecke: Solid-State Electron., 18 (1975) 1146. 